\title{
WEB BASED ACCESS CARD GENERATION USING FACE RECOGNITION: A TECHNICAL REVIEW
}

\author{
Dhairyaraj Ataliya, Krunal Chauhan, Kuldeep Chotaliya, Kevin Thoriya \\ Student, Department of CSE, Parul Institute of Technology, Parul University, Vadodara, Gujarat, India \\ Rumana Shaik \\ Assistant Professor, Department of CSE, Parul Institute of Technology, Parul University, Vadodara, \\ Gujarat, India
}

\begin{abstract}
Right now the world contributes significant jobs in all constant frameworks. Different administration frameworks executed for accomplishing the business association towards benefits, principles, and further business improvement. Records are snippets of data made or got by an association or business foundation that gives confirmations of business choices or exchanges. Record the board is an imperative piece of any association remembering the day by day records of guests for the association. Kinds of records incorporate crucial and nonindispensable records, fundamental and insignificant records, dynamic and non-dynamic records, and so forth a portion of these classes of records are required very quickly to continue back the running and activity of an association if there should be an occurrence of a crisis, accordingly, they ought to be safely put away and overseen. Strangely, these records could be put away in paper-based organizations or a computer-based based framework. A visitor management system is a method for handling visitor information on-campus visits. Any company that holds the visitor's data is a very big issue. This system would replace the manual maintenance process and help to keep track of visitors to the campus by the authorities. For this manual entry replacement, we propose an automatic access card generation system "VISNET". This system helps for visitor seamless check-in and check-out, the visitor knows basic details of his purpose with message/mail. This system will replace the manual maintenance system and can help the authorities to stay track of the visitors visiting the campus. This software will help a corporation to try to do the work effectively and provides an all-round development of the project.
\end{abstract}

Keywords - E-Mail, Face recognition, OTP, QR code

\section{INTRODUCTION}

The visitor management system is usually used in places where a large number of visitors come and go. It becomes difficult to keep track of all the visitors by a few employees. VISNET provides an easy method for the front desk officer to search for the ongoing visit of the day. They will be timely notified of this visitor visiting their department. The searching method is also faster and the system will give output that the user needs. Currently, most of the organization is using the named method in keeping track of all the incoming and outcoming visitor records in each of the departments. The problem arose when at a certain point in time the number of visitors visiting the department increased and unable to manage and messed up. On top of that, a security issue also is the main issue in generating visitor's pass in that particular organization. Furthermore, the log register has been accustomed to track all the visitor records and it's not enough. Log register isn't an efficient method to keep records in several years, and so as to supply reports, retrieve or inquiry previous records especially 2-3 years back is extremely hard to urge and takes tons of your time to retrieve the records and reports. VISNET will generate a Photo ID of the first-time visitors in softcopy format. This is stored within the database to take care of records of all the people that visited the building. Then print the Photo ID for one-time or continuing use to hurry the safety screening process. Modern visitor management systems enable the operator to understand the entire visitors inside the premises. VISNET also helps users access information inquiry faster. Compared to the manual system, the user needs to go page by page to search for information. Imagine there were hundreds of visitors coming in and out. However, with the new system, the user only needs to search via card number and as a result, the system will display related output that was entered by the user earlier. A problem such as waiting for a long-time queue will be no more a problem and will give an impression of a well-organized system. 


\section{OBSERVATION}

\section{A. QR based Technology ${ }^{[1]}$}

The author gave us an appropriate introduction to the $\mathrm{QR}$ code. First of all, he gave the knowledge of different versions of the QR code and the latest version is used. After that, he gave the introduction of different segments of the QR code and gave a short introduction about that. Then he acknowledges us about "Reed-Solomon codes" which are used for error-correction in the QR code. additionally, it gave the error-correction level and percentage of correction. The structure of the QR code helps us to know different regions of QR code like function pattern, encode region, Quite zone. How QR code encoded and where information was stored. At last, he shows how the scanner scans QR code and retrieves the information and what are the different types of $Q R$ codes that are currently in use.

\section{B. Increase capacity of $\mathbf{Q R}$ code ${ }^{[2]}$}

This paper proposed a new method for increasing the storage capacity of QR code with the help of colored QR code. Traditional QR code stores the 1-bit information in one module where this type stores 4-bit data in that. This method follows the traditional approach with a slight change in the color model. For that, it uses an HSV (Hue, Saturation, Value) color model. For the decoding process, it uses the Visual Studio .NET Compact Framework environment with C\# on HTC Touch Diamond phone and Windows Mobile 6.1 phone. Both were equipped with a 3.2-megapixel camera. They found that the alignment of color code enhances the quality of the decoding process and also increases the data capacity. Both encoding and decoding processes are developed on the Java platform. We have to use an 8-megapixel camera for better accuracy. This was the limitation of this application and also this whole model developed on the JAVA based platform.

\section{Expression negation algorithm ${ }^{[3]}$}

In this paper, the authors focus on the most challenging problem in face recognition, when there is less number or only one of the training images available, the problem known as a single sample per person problem. He proposes an algorithm based on removing facial expression from the expression face image. In that, they prepare face models based on a facial expression like happy, neutral, surprise, disgust, etc. The algorithm goes like first they capture an image from the system then wrap the image to their corresponding expression face model. The algorithm removes expressions and obtains the synthesized neutral image. It removes the dimensions from the synthesized neutral image by applying principal component analysis and classifies it using a cosine similarity measure.

\section{$D$. Intra-class facial variation method ${ }^{[4]}$}

This proposed paper focuses on a single sample training image for facial recognition. This novel approach mainly focuses on intra-class facial variation with that it expands the training sample. In that first, the different images of face data-based are used to minus their corresponding neutral expression face to obtain intra-class facial variation with the same other intraclass variation images to calculate the average intra-class variation with that generated intra-class facial variation set. The procedure is to get the neutral image of a person using this image. It can expand the training set using the average intra-class facial variation set. At last, both the expanded database and primary database are compared.

\section{E. Trend of QR technology ${ }^{[5]}$}

In this proposed paper the author gave the information of various areas where the use of QR code grows exponentially. She also gave information, how the Organization uses QR code in different sectors like the Tourism department, Airports, Article writers, banking and payments, education, mobile apps, product and packaging, and cybermedia etcetera.

\section{$F$. Identification card-based visitor management system ${ }^{[6]}$}

In this proposed paper researcher gave a new way to replace the traditional visitor management system. This approach system uses country unique identification cards to get access to user details. With that, they fetch basic details. According to the details, the system is permitted to enter premises. The main flaw of the system is when the user does not want to give access to their access card information then the system is not permitted to enter premises.

\section{G. Comparison of web development framework ${ }^{[7]}$}

This proposed paper gave us the practical comparison of Django, Ruby on Rails and CakePHP on the various aspects like user interface development, maintainability, data management and migration, testability, popularity, community and maturity, and marketability. With the practical evolution of all the given parameters, the total score of Django 4.05, Ruby on Rails 3.85, CakePHP 2.95. From this score, we can say that Django is the best web development framework among them.

\section{H. LCD display based system ${ }^{[8]}$}

This research paper is based on guiding a visitor to its desired location in a building with the help of signs and symbols. An automated guidance system for guiding an individual to a particular region includes an access control mechanism containing a selectively encoded readable feature in one dimension of the invention. An access control system reader reads and rejects the encoded readable item, or enables a user to enter a reader- predetermined position. A communication 


\section{International Journal of Engineering Applied Sciences and Technology, 2020 \\ Vol. 4, Issue 12, ISSN No. 2455-2143, Pages 379-384 \\ Published Online April 2020 in IJEAST (http://www.ijeast.com)}

system used with the application reader for access control sends a notification that guides the user to the predetermined position when the user is enabled to reach the predetermined position managed by the reader.

\section{Analysis of Visitor management activities ${ }^{\text {[9] }}$}

In this research paper we studied Visitor management activities like card issue /return, visitor check-in/check-out for entry into the secure areas of large organizations, which are usually done by an operator (e.g., a receptionist) at the reception desk. The main concern about the proposed idea is Often a pass is issued that provides access to the secure area by the visitor. In some cases, a great deal of time may be lost waiting for passes or contacting the proper person within the organization.

\section{J. Django The Python Web Framework ${ }^{[10]}$}

This proposed paper was used to demonstrate the features of Django. Django is a high-level Python framework by using it the development speed will be faster and cleaner. Various web application frameworks use MVC (Model-View-Controller) development. Model-view-controller is the pattern of the web application framework in which models and views are defined to make interconnection with data and controllers are used for defining routes and templating. But Django has done all controller work on its own. So, Django works on the MTV(Model-Template-view) model by which developers write business logic. The main advantage of Django is based on python and it has characteristic features like it is Interactive, interpreted, Modular, Dynamic, Object-oriented, Portable. Django makes the event process remarkably quickly, from idea to release through to launch and production. With transparent, clean code, development is often both efficient and effective. Django is fast, secure and flexible. Django is to take the developers from project conception to the launch platform quickly, and it helps in that. It also takes away the burden of security issues like cross-site request forgery, SQL injection, cross-site scripting, and clickjacking. Developers can manage user accounts and passwords through a reliable user authentication system. It delivers transparent and highquality code writing, making it important for developers, and equally important for patrons.

\section{$K$. Face Recognition Using Eigenface Approach ${ }^{[11]}$}

In this research paper authors explain the PCA based algorithm. They explained how the algorithm is going to be implemented. This algorithm is based on an eigenface approach. They said that it is based on different features of faces. They used a PCA based eigenface algorithm for the improvement of a face recognition system. They said that this algorithm converts faces into a set of characteristics, eigenfaces which are the main part of the training dataset. The recognition part can be done through overlapping new faces on the eigenface. It is very fast and provides simplicity to the systems. There is only a problem that faces should be in the vertical view of the human face. It helps us to know about the PCA based eigenfaces algorithm. Then they talked about how the PCA based approach is used for face recognition. At last, they explained to us about the success rate of the algorithm. They said that it is based on training images of the person.

\section{Face Recognition Using PCA \& LDA Algorithms ${ }^{[12]}$}

The authors explain what face recognition is, how face recognition can be done? why face recognition is important. It also explain the different types of algorithms. They compare other biometric technology with face recognition. They explain that password-based systems are easy to hack and face recognition systems are hard to hack. They explain that many factors are important in face recognition like Intensity, pose, structural components, image rotation, and poor quality. They explain two algorithms: PCA (Principle Component Analysis) and LDA (Linear Discriminant Analysis). They explain that PCA is a statistically reduced method which uses eigenfaces for it. It is used for data compression of the photo dimension. This algorithm removes unnecessary data of the face and stored as eigenface. It only stored the necessary data. LDA is a data separation technique for face recognition. LDA is based on separating the face data into a different class. Both demand low memory, low computational complexity. In the end, they said that the Face recognition system is better than any previous biometric system. It helps us to know about the LDA and PCA based algorithms.

\section{Face Recognition Techniques and Approaches: A Survey ${ }^{[13]}$}

In this paper, the authors explain about the different approaches and techniques of face recognition. They explain how people's faces are recognized in the system. They said that face recognition systems can be divided into three parts: Face detection, Face segmentation and Face recognition. They also explain the algorithms like LDA (Linear Discriminant Analysis), PDA (Principle Component Analysis) and EBGM (Elastic Bunch Graph Matching). PCA is used for solving a single sample per person's problems. LDA is used for dimensionality reduction of feature extraction. They also explain different face recognition approaches like the Holistic approach, Statistical approach, Hybrid approach, Featurebased approach, and model-based approach. A holistic approach considers the whole face as a single feature. It ignores single features like an eye, ear, nose, etc. The featurebased approach considers the whole face as well as a single feature like eye, ear, nose. The model-based approach is used for $3 \mathrm{D}$ face recognition. It helps us to know about different approaches and methods of face recognition. In the end, they 
conclude that a hybrid-based approach is best for facerecognition.

\section{$N$. Deep Face Recognition ${ }^{[14]}$}

The Authors explain about deep face recognition. They explain this algorithm that they need two things for the algorithm: Single photograph or set of face tracked video and very large dataset. They used CNN (Convolutional Neural Network) for the training of the dataset.it helps us to know about the new deep face algorithm.

\section{$O$. Recognition of an Individual using the Unique Features of Human Face ${ }^{[15]}$}

In this proposed paper the authors explain about LDA based algorithms. They also talk about face recognition steps. They explain that PCA is a holistic based approach which calculates eigenfaces based on eigenvectors. PCA can be done in four steps: Facial image acquisition, image pre-processing, feature extraction from facial images, Principal component analysis. This paper focuses on how PCA works. It helps us to know about how PCA can be done. They first do normal PCA then they filtered some eigenfaces with the use of minimum Euclidean distance. They explain the filtered faces used in the second training of the dataset. In the end, they said that their approach can produce more accurate results than the normal PCA algorithm.

\section{$P$. Authentication Protocols based on OTP ${ }^{[16]}$}

In this proposed paper the author explained about different OTP generation mechanisms as well as advantages and disadvantages of mechanism against various networking attacks. Several OTP techniques are introduced by the end of 2013 like Simple hash function protocol, Lamport's Hash Chain Protocol, YSH Protocol, Bicakci Protocol Which is not applicable for the present because of the disadvantage and its low resistance against various attacks in this modern world. They briefly explain that nowadays Chefranov's, HOTP, TOTP mechanisms are used for OTP generation because of their advantage and high security against various attacks.

\section{$Q$. Analysis of OTP Generation Techniques ${ }^{[17]}$}

In this proposed paper the author gave different types of OTP generation techniques and how they generate the OTP. TOTP (time synchronization OTP) While sharing a secret key, client and server synchronized Time. After generating the OTP by secret key and time sent to a server. the server computes its OTP with its time and shared secret key. both OTP matched then the user can access data. This OTP can be used multiple times too only in that time frame. HOTP (Hash or Event synchronization OTP) The server initializes one counter while sharing a secret key. The client generates an OTP with a counter and secret key and sends it to the server. The server utilizes the same algorithm with a counter and secret key to generate OTP. If both OTP are the same then the user can access the data. OTP can be used only for one time for a second time counter value is incremented by OCRA (Oath challenge-response Authentication) In this technique, the server authenticates a user using challenge values. When the client wants to communicate, the server sends the challenge based on the secret key and every time the challenge values are different. The client now generates an OTP based on a challenge message and shares a secret key and sends it to the server. The same task performed by the server to generate OTP. If both are the same then the user is authenticated. This OTP can be valid once only. for second-time challenge values are different.

\section{$R$. Study on Providing Reliable and Secure SMS Authentication Service ${ }^{[18]}$}

The author analyses the problem of the conventional SMS authentication method and presents a secure SMS authentication method using a mobile phone. Secure authentication is made possible by adding two steps. A user recognizes and verifies the SMS on arrival, and then the user confirms again after the initial authentication so It can be safely used in the payment system such as in micropayment. The main problem in the conventional SMS authentication method is mutual authentication and non-repudiation of the payment system. The Secure SMS authentication method provided security services as confidentiality, integrity, nonrepudiation, and mutual authentication. Authentication is done in 3 steps as follows. Registration Step- In the registration process, the certificate and stored key, a user installs an additional program on his mobile phone and sets a password to the program password. This program includes the decryption of authentication text messages and the capability to generate the digital signature. Utilization StepThis step will generate the authentication information, encrypt it with a public key (generate while registration) and digital signature. Verification Step- The program decrypts the corresponding text message by using the certificate (user private key), which is stored in the user's mobile phone, or using the symmetric key, and verifies the signature value (the mobile communication carrier's public key). If the signature is correct, the program displays the authentication text message.

\section{S. Real-Time Face Recognition of Human Faces by using LBPH and Viola Jones Algorithm ${ }^{[19]}$}

In this paper, the authors have provided information about two algorithms that are used for face recognition. The two algorithms are the LBPH algorithm and Viola Jones algorithm. They said that the face recognition system has three phases which are Face detection, Feature extraction and 
Face recognition. They do face recognition using the Viola Jones algorithm and features extracted by LBPH. First, they collect face data. Then they do recognition using the viola jones algorithm. Then they extracted features using LBPH. They said that LBPH (local binary pattern histogram) is a collection of binary values of pixels. It helps us to know about LBPH and viola jones algorithm. At last, they said that using this algorithm they achieve $85 \%-95 \%$ accuracy.

\section{T. Web Application Security and Detecting Security Vulnerabilities ${ }^{[20]}$}

Authors state that web security is the biggest challenge in the corporate world. It is considered as the principle framework for the worldwide data society. Web applications are prone to security attacks. Web security is securing a web application layer from attacks by unauthorized users. A lot of the issues that occur over a web application is mainly due to the improper input provided by the client. The main elements of web security techniques such as passwords, encryption, authentication, and integrity. There are two common important security vulnerabilities today: SQL injection and cross-site scripting. These types of vulnerabilities directly affect web servers, application servers, and web application environments. Paper covered all properties of web application development, understood the important security functions and properties that secure web applications should use and divided existing works into three major classes. and discuss a few issues that still need to be considered. To access a few out of the box features in web applications various programming concepts and tools are taking place that cause essential security aspects to our applications.

\section{PROPOSED METHODOLOGY}

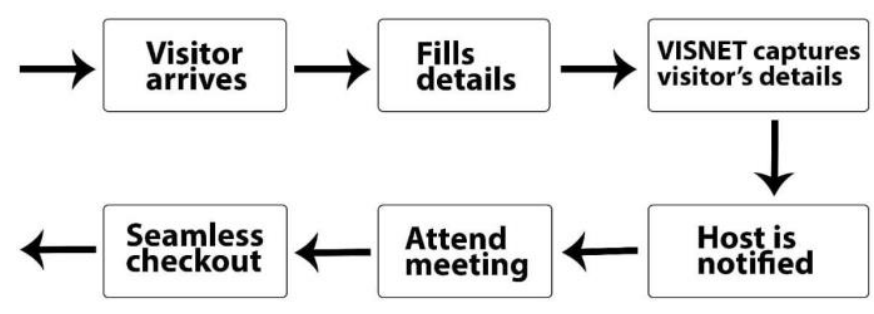

Fig. 1 VISNET Work Mechanism Flowchart

The figure shows the Work Mechanism flow of the system.

Online - In WebApp, the registration link will be provided on the site of the organization or the registration desk. From the visitor, we get information like Name, Phone number, Email, Purpose of visit, etcetera. Also, take phone numbers for authentication purposes, OTP will be sent to that mobile number. Then the visitor adds the purpose of their visit and then QR code will be sent to his/her email address. When they come to the organisation then they scan it at the main gate of an organisation and get the access card. If the user comes to visit again on premises, the system will be featured with Face recognition. For face recognition, we use LBPH algorithm. In the face recognition process, they don't want to enter the information. They simply scan their face and information can display. They simply enter the purpose of the visit and access cards can be generated.

Offline - For offline purposes, they simply come to a premise and enter the information and generate the access card. All other processes will be the same.

\section{CONCLUSION}

There has been continuous advancement in science and technology. The latest technology and gadgets are being designed and developed. This web application can be used inside any campus for automatic entry of visitors accessing and departing for the campus. As discussed formerly, in this project we have created a system whose main objective is to provide automatic access cards without much human effort. We have used different modules to create a secure system. Face recognition will be used to retrieve information using previous records. OTP will be generated for Visitor authentication. QR Code is used to store user's information and can be used to log out from the system. The system created is efficient, reliable and has a promising performance. In the future, with the advancement of new technology few improvements will be essential to continue the usability of the system.

\section{REFERENCE}

[1] Tiwari, S. (2016, December). An introduction to QR code technology. In 2016 International Conference on Information Technology (ICIT) (pp. 39-44). IEEE.

[2] Taveerad, N., \& Vongpradhip, S. (2015, November). Development of color QR code for increasing capacity. In 2015 11th International Conference on Signal-Image Technology \& Internet-Based Systems (SITIS) (pp. 645648). IEEE.

[3] Tharshini, G., Dinesh, H. G. C. P., Godaliyadda, G. M. R. I., \& Ekanayake, M. P. B. (2015, December). A robust expression negation algorithm for accurate face recognition for limited training data. In 2015 IEEE 10th International Conference on Industrial and Information Systems (ICIIS) (pp. 384-389). IEEE.

[4] Zeng, J. Y., Zhao, X. X., Zhai, Y. K., Gan, J. Y., Lin, Z. Y., \& Qin, C. B. (2017, July). A novel expanding sample method for single traning sample face recognition. In 2017 International Conference on Wavelet Analysis and Pattern Recognition (ICWAPR) (pp. 33-37). IEEE. 


\section{International Journal of Engineering Applied Sciences and Technology, 2020 \\ Vol. 4, Issue 12, ISSN No. 2455-2143, Pages 379-384 \\ Published Online April 2020 in IJEAST (http://www.ijeast.com)}

[5] Dyade M, Bhande Y. (2017). Use of QR Code: A Step Towards Development in India. International Research Journal of Engineering and Technology, 4, 1165-1168.

[6] Oktaviandri, M., \& Foong, K. K. (2019). Design and Development of Visitor Management System. Mekatronika, 1(1), 73-79.

[7] Plekhanova, J. (2009). Evaluating web development frameworks: Django, Ruby on Rails and CakePHP. Institute for Business and Information Technology.

[8] Kshirsagar, S. R., Subbaiah, R., \& Subbiah, S. (2009). U.S. Patent No. 7,600,679. Washington, DC: U.S. Patent and Trademark Office.

[9] Muthupandian, A. V. S., \& Venkatesan, B. (2013). System and Method For Automatic Visitor Check-In And Access Card Issuance, U.S. Patent Application No. 13/462,675.

[10] Ramesh, P. B. N., Amballi, A. R., \& Mahant, V. (2018). DJANGO THE PYTHON WEB FRAMEWORK. International Journal of Computer Science and Information Technology Research, 6, 59-63.

[11] Slavković, M., \& Jevtić, D. (2012). Face recognition using eigenface approach. Serbian Journal of electrical engineering, 9(1), 121-130.

[12] AbhishekBansal, K. M. (2012). SahilArora, "Face Recognition Using PCA \& LDA Algorithms". IEEE Computer Communication and Informatics, 1-5.

[13] Naeem, M., Qureshi, I., \& Azam, F. (2015). FACE RECOGNITION TECHNIQUES AND APPROACHES: A SURVEY. Science International, 27(1).

[14] Parkhi, O. M., Vedaldi, A., \& Zisserman, A. (2015). Deep face recognition.

[15] Matin, A., Mahmud, F., \& Shawkat, M. T. B. (2016, December). Recognition of an individual using the unique features of human face. In 2016 IEEE International WIE Conference on Electrical and Computer Engineering (WIECON-ECE) (pp. 57-60). IEEE.

[16] Babkin, S., \& Epishkina, A. (2019, January). Authentication Protocols Based on One-Time Passwords. In 2019 IEEE Conference of Russian Young Researchers in Electrical and Electronic Engineering (EIConRus) (pp. 1794-1798). IEEE. Huang,

[17] Y., Huang, Z., Zhao, H., \& Lai, X. (2013). A new onetime password method. IERI Procedia, 4, 32-37.

[18] Lee, J., \& Oh, Y. (2014, December). A study on providing the reliable and secure SMS authentication service. In 2014 IEEE 11th Intl Conf on Ubiquitous Intelligence and Computing and 2014 IEEE 11th Intl Conf on Autonomic and Trusted Computing and 2014 IEEE 14th Intl Conf on Scalable Computing and Communications and Its Associated Workshops (pp. 620624). IEEE.
[19] Suma, S. L., \& Raga, S. (2018). Real Time Face Recognition of Human Faces by using LBPH and Viola Jones Algorithm. International Journal of Scientific Research in Computer Science and Engineering, 6(5), 610.

[20] Kumar, S., Mahajan, R., Kumar, N., \& Khatri, S. K. (2017, September). A study on web application security and detecting security vulnerabilities. In 2017 6th International Conference on Reliability, Infocom Technologies and Optimization (Trends and Future Directions)(ICRITO) (pp. 451-455). IEEE. 